\title{
Mecanismos moleculares implicados en la destrucción ósea en la periodontitis. Revisión de la literatura
}

\author{
Molecular mechanisms involved in bone destruction in \\ periodontitis. Literature review
}

Moreno Correa $\mathrm{S}^{1}$, Contreras Rengifo $\mathrm{A}^{2}$

\begin{abstract}
RESUMEN
La periodontitis es una enfermedad inflamatoria crónica multifactorial, la cual se inicia a partir de la biopelícula que se forma alrededor de los dientes y se acumula en margen gingival, colonizando el surco gingival. La complejidad de la biopelícula madura genera estímulos para las células epiteliales e inflamatorias y sobre las demás células del tejido conectivo activando los mecanismos de la respuesta inmune innata y adaptativa. Se reconoce que la acumulación de placa dental genera de forma indefectible gingivitis, pero se desconocen las señales específicas que disparan la periodontitis. Se reconoce también que microorganismos periodontopáticos como Aggregatibacter actynomicetemcomitans, Porphyromonas gingivalis, Tannerella forsythia, Treponema denticola, Prevotella intermedia, Prevotella nigrescens, entre otros, poseen mediadores osteolíticos que actúan directa o indirectamente en las células del hueso y que son responsables del proceso de remodelación ósea, lo cual desequilibra el eje RANKL-RANK/OPG. Los productos microbianos y la respuesta inflamatoria inducen la secreción de citoquinas específicas como IL-1B, TNFa y otros mediadores pro-inflamatorios como PGE $_{2}$, metalloproteinases, MMP-8, MMP-3, RANKL, además los linfocitos $T$ y $B$ activados inducen la pérdida de hueso alveolar al sintetizar y secretar directamente RANKL. Debido a que la pérdida de hueso alveolar es uno de los signos patognomónicos de la enfermedad periodontal, se hace importante revisar los mecanismos moleculares que explican la destrucción ósea, así como algunos avances en el tratamiento óseo.
\end{abstract}

Rev. Clin. Periodoncia Implantol. Rehabil. Oral Vol. 6(3); 142-147, 2013.

Palabras clave: Periodontitis, destrucción ósea periodontal, terapia periodontal.

\section{ABSTRACT}

Periodontitis is a multifactorial chronic inflammatory disease started by biofilm accumulation around the teeth and the gingival margin including the gingival sulcus. Mature biofilm is complex in microbial nature and it triggers signals to the boundary connective tissue and epithelial cells activating mechanisms of innate and acquired immune response. It is known that the dental plaque accumulation indefectibly results in gingivitis. However the specific signals that lead to periodontitis are unknown. The main periodontopathic organisms are Aggregatibacter actynomicetemcomitans, Porphyromonas gingivalis, Tannerella forsythia, Treponema denticola, Prevotella intermedia, Prevotella nigrescens among others. Those microorganisms produce osteolytic mediators that act directly and indirectly on bone cells affecting the bone turnover rate, regulated by the axis RANKL-RANK/OPG. Microbial products and periodontal inflammation induce the release of specific cytokines IL-1B, TNFa, PGE2, metalloproteinases, MMP-8, MMP-3, RANKL, T and $\mathrm{B}$ lymphocytes elicit bone resorption. Indeed, alveolar bone loss is one of the most pathognomonic features of periodontal disease. Therefore it is essential to review the molecular mechanisms explaining periodontal destruction, as well as the advances in bone therapy.

Rev. Clin. Periodoncia Implantol. Rehabil. Oral Vol. 6(3); 142-147, 2013.

Key words: Periodontitis, periodontal bone destruction, periodontal therapy.

\section{INTRODUCCIÓN}

La periodontitis es una enfermedad inflamatoria crónica que afecta los tejidos de soporte y protección del diente como son la encía, el ligamento periodontal y el hueso alveolar, resulta de la acumulación de la biopelícula bacteriana alrededor del margen de la encía y posiblemente del sobrecrecimiento de patógenos periodontales específicos ${ }^{(1-3)}$.

La enfermedad periodontal crónica es una de las 7 patologías periodontales propuestas por la Asociación Americana de Periodoncia en 1999 y es la más prevalente en pacientes adultos. La periodontitis crónica se clasifica a su vez en localizada o generalizada, según la extensión de la enfermedad, y en leve, moderada o avanzada, según la severidad que se relaciona con el nivel de pérdida de inserción clínica y la profundidad al sondaje de la bolsa periodontal. En una periodontitis crónica leve hay una pérdida de inserción de 2 a $3 \mathrm{~mm}$, de 3 a $4 \mathrm{~mm}$ en la periodontitis crónica moderada y más de $5 \mathrm{~mm}$ en la periodontitis crónica severa ${ }^{(4)}$

Clínicamente el nivel de pérdida de inserción se evidencia por la migración apical de los tejidos periodontales y exposición radicular, también podemos observar signos clínicos como la inflamación, el sangrado al sondaje y en algunos casos la supuración. Radiográficamente se observa la pérdida de hueso alveolar y la pérdida de soporte del diente, lo cual, en los casos más severos, conlleva a la pérdida dental.

La periodontitis crónica tiene una alta prevalencia. En Colombia, según el III Estudio Nacional de Salud Bucal en 1999(5), se encontró una prevalencia del $55 \%$ en el nivel de pérdida de inserción. En el mundo, la prevalencia es en promedio del 33\%, siendo en algunos países más prevalentes que en otros, aunque estos estudios epidemiológicos han sido complejos debido a las variaciones en las definiciones y en la clasificación de las patologías periodontales ${ }^{(6)}$.

En la etiopatogénesis de la enfermedad periodontal, la participación de los microorganismos es esencial, pero se han considerado insuficientes para explicar la destrucción periodontal, es por esto que factores del huésped como son la respuesta inmune, factores ambientales como fumar, factores genéticos como ciertos polimorfismos de citoquinas y factores sistémicos como la diabetes, son igualmente importantes y determinantes para que se desarrolle la enfermedad.

1. OD, MSc. Grupo de Medicina Periodontal. Escuela de Odontología, Universidad del Valle. Cali-Colombia.

2. OD, MSc, PhD. Grupo de Medicina Periodontal. Escuela de Odontología, Universidad del Valle. Cali-Colombia.

Correspondencia autor: Sandra Moreno. sandramimoreno@yahoo.com. Trabajo recibido el 15/02/2012. Aprobado para su publicación el 05/05/2012. 
Muchos autores se han enfocado en esta compleja interacción y han catalogado a la enfermedad periodontal como una enfermedad multifactorial, la cual también está implicada en diferentes complicaciones sistémicas, como la enfermedad cardiovascular, la aterosclerosis, la preeclampsia, el parto prematuro y el bajo peso al nacer, entre otras ${ }^{(7,8)}$.

Debido a que la pérdida de hueso alveolar es un proceso importante dentro de la enfermedad periodontal y es la que conlleva a la pérdida dental, conocer los mecanismos moleculares implicados en éste es muy importante desde el punto de vista de progresión de la enfermedad, como también para establecer opciones de tratamiento.

El hueso es un tejido conectivo especializado mineralizado y altamente vascularizado e inervado, cuenta con diferentes tipos celulares, dentro de los cuales se encuentran dos tipos de células que participan en el proceso de remodelación ósea como son el osteoblasto y el osteoclasto.

El osteoblasto es una célula que se encarga de sintetizar y secretar proteínas de matriz extracelular de hueso, mineralizar la matriz y regular las funciones del osteoclasto. El osteoclasto, a su vez, se encarga del proceso de resorción ósea ${ }^{(9,10)}$

\section{CÉLULAS Y MECANISMOS MOLECULARES IMPLICADOS EN EL PROCESO DE REMODELACIÓN ÓSEA}

\section{Osteoblasto: Estructura y Función}

El osteoblasto es una célula grande, de forma poliédrica ,que se caracteriza por tener un retículo endoplásmico y un aparato de Golgi grandes. Proviene de células mesenquimales y tiene una vía de diferenciación similar a la de los adipocitos y condrocitos, se diferencia de éstos por expresar los factores de transcripción Runx 2 (cbfa 1) y osterix. Esta célula se encarga de formar hueso en dos fases, la primera consiste en sintetizar y secretar proteínas de matriz extracelular de hueso como son osteonectina, osteopontina, sialoproteína de hueso, colágeno tipo I, glicoproteínas y otras proteínas de matriz. La segunda fase consiste en la mineralización de esa matriz extracelular y lo hace por la expresión y secreción de la enzima fosfatasa alcalina. Esta célula también secreta factores de crecimiento como son el factor de crecimiento transformante $\beta$ (TGF- $\beta$ ), factor de crecimiento tipo insulina I y II (IGF I y II), factor de crecimiento básico de fibroblastos (bFGF) y proteínas morfogénicas de hueso (BMP), los cuales actúan de forma autocrina en la misma célula activando su función, por lo tanto en los procesos de formación de hueso, estos factores están ampliamente expresados ${ }^{(11,12)}$.

\section{Osteoclasto: Estructura y Función}

El osteoclasto es una célula gigante multinucleada que posee un retículo endoplasmático y un aparato de Golgi bien desarrollados, abundantes lisosomas, vesículas y mitocondrias pleomórficas. Se caracteriza por presentar dos especializaciones de membrana que son los bordes en cepillo y las zonas claras.

Las zonas claras tienen abundantes microfilamentos de actina y expresan una integrina denominada $a b \beta 3$, la cual se une con una alta afinidad a secuencias de aminoácidos Arginina, Lisina y Aspartato de proteínas de matriz ósea como osteopontina y sialoproteína de hueso. Esta fuerte unión permite que se selle el área de resorción que se encuentra debajo de los bordes en cepillo y que se conoce como laguna de Howship's.

En la membrana de los bordes en cepillo se encuentra una AT pasa de hidrógeno que bombea protones hacia el interior de la laguna de Howship's; concomitantemente se secretan iones de cloro a través de canales de cloro, esto crea un ambiente lo suficientemente ácido como para permitir la disolución de los minerales del hueso. El osteoclasto también secreta enzimas lisosomales que degradan la sustancia orgánica del hueso y cathepsina $\mathrm{K}$ y metaloproteinasa de matriz 9 (MMP-9) las cuales se encargan de degradar el colágeno tipo I. La célula también expresa y secreta la enzima fosfatasa ácida tartrato resistente (TRAP), la cual degrada proteínas de matriz de hueso; esta enzima se puede marcar con inmunohistoquímica y sirve como un marcador de identificación de osteoclastos tanto In Vivo como In Vivo.

Productos de degradación de hueso son endocitados a través de la porción central de los bordes en cepillo por vesículas trancitoticas y son exocitados en el dominio secretor funcional.

Esta célula también expresa ampliamente receptores de calcitonina, la calcitonina es una enzima que inactiva el proceso de resorción ósea en el osteoclasto y expresa una proteína de membrana de 7 hélices $a$ denominada DC-STAMP, la cual permite una unión fuerte entre osteoclastos precursores uninucleares que conduce a su fusión y diferenciación en un osteoclasto multinucleado ${ }^{(11-13)}$.

El osteoclasto proviene de línea hematopoyética del linaje monocito/macrófago, estas células progenitoras se diferencian en un osteoclasto precursor y estas células precursoras se unen fuertemente por la interacción de la proteína DC-STAMP lo que conduce a la fusión de estas células y diferenciación hacia una célula multinucleada la cual se puede diferenciar hacia un osteoclasto activado que presenta la morfología antes descrita. Esta diferenciación se da gracias a la interacción de ciertas citoquinas, que en el tejido óseo produce y secreta principalmente el osteoblasto(14-16).

\section{Regulación de la Diferenciación y Función de Osteoclastos}

Debido a la acción de ciertos estímulos, como son la 25-dihydroxyvitamin D3 $\left(1.25(\mathrm{OH})_{2} \mathrm{D}_{3}\right)$, Prostaglandina $\mathrm{E}_{2}\left(\mathrm{PgE}_{2}\right)$, Hormona paratiroidea (PTH), IL-11, etc., el osteoblasto secreta la citoquina Factor Estimulante de Colonias de Macrófagos (M-CSF), la cual se une a receptores c-Fms que expresa el osteoclasto precursor; la activación de éste receptor activa una vía de señalización que conduce a la diferenciación hacia osteoclastos u osteoclastogénesis ${ }^{(13)}$

El osteoblasto también secreta una citoquina de la familia del Factor de Necrosis Tumoral a (TNF- $\alpha$ ), denominada Receptor Activador del Factor Nuclear $K \beta$ Ligando (RANKL), esta se une al receptor RANK que expresan los osteoclastos precursores y los osteoclastos diferenciados, la unión entre RANKL-RANK induce diferenciación, activación y supervivencia de osteoclastos.

Como un mecanismo de regulación el osteoblasto secreta un receptor soluble de la familia de los receptores del TNF- $\alpha$, denominado Osteoprotegerina (OPG), la cual se une a RANKL y bloquea su unión con RANK(17).

La unión de RANKL con su receptor RANK en los osteoclastos precursores u osteoclastos diferenciados conduce a la unión de la porción citoplasmática de RANK con una proteína adaptadora denominada TRAF6 lo cual activa una vía de señalización que activa el Factor Nuclear K $\beta$ (NFK $\beta$ ) y esto permite la expresión del Factor de Transcripción de Células T Activadas Citoplasmático 1 (NFATc1), el cual es el factor de transcripción maestro del osteoclasto. Para que se de la osteoclastogénesis, este factor de transcripción no solo necesita expresarse sino también activarse y para esto requiere su translocación al núcleo, y esta activación se da gracias a la acción de la calcineurina, una hormona que es activada por la movilización del calcio intracelular que se genera por la interacción entre RANKL-RANK y es regulada por la proteína $\mathrm{G}$ de membrana RGS10. Para que se dé una respuesta robusta y eficiente se requieren señales coestimuladoras que son posibles gracias a la expresión del Receptor Activador de Osteoclastos (OSCAR) y el receptor TREM-2 por el osteoclasto los cuales son activados por ligandos que posiblemente son producidos por el osteoblasto pero que se desconocen hasta la fecha. La activación de estos dos receptores conlleva a la unión con dos proteínas adaptadoras como son fcRy y DAP12, lo cual activa vías de señalización que conllevan a una mayor movilización del calcio intracelular, mayor activación de calcineurina y la potenciación en la activación del factor de transcripción NFATc1 lo cual conduce a una respuesta positiva y eficiente que en este caso es la osteoclastogenesis ${ }^{(18)}$.

El eje RANKL-RANK/OPG guía el metabolismo del hueso y los procesos de remodelación ósea, lo cual permite el crecimiento del esqueleto y la capacidad de autoregenerarse después de un trauma ${ }^{(13)}$.

Debe existir un equilibrio entre la secreción de RANKL y OPG para mantener la homeostasis del tejido, de tal manera que la cantidad de hueso que se reabsorbe sea igual a la cantidad de hueso que se forma, por lo tanto en los procesos de patología se genera un desequilibrio o disbiosis donde se produce una gran cantidad de RANKL comparado con poca cantidad de OPG lo cual conduce a un aumento de la resorción ósea y el hueso que se forma es insuficiente.

En la enfermedad periodontal se da lugar este desequilibrio lo cual conduce a la pérdida de hueso alveolar ${ }^{(19)}$.

Mediadores Osteolíticos Bacterianos en la Enfermedad Periodontal (Tabla 1)

La biopelícula bacteriana presente en la enfermedad 
periodontal está compuesta por una gran cantidad de microorganismos, aproximadamente 500 especies que interactúan y que están constantemente expresando sus factores de virulencia y mediadores, induciendo diferentes respuestas en el huésped y desencadenando el daño en el tejido óseo y periodontal(2).

El Lipopolisacarido (LPS) es un componente de la membrana externa de las bacterias Gram negativas, es un factor de virulencia importante por su capacidad de desencadenar la respuesta inflamatoria ${ }^{(20)}$ también actúa como un mediador osteolítico por cuatro vías diferentes que estimulan, primero, la producción de RANKL directamente en el osteoblasto, segundo producción de Prostaglandina $E_{2}\left(P E_{2}\right)$ por el osteoblasto, tercero la diferenciación y activación de osteoclastos y cuarto la producción de citoquinas pro-inflamatorias ${ }^{(21)}$ (Figura 1).

El primer mecanismo es posible gracias a que el osteoblasto expresa el receptor tipo Toll IV (TLR4) y su co-receptor CD14, los cuales reconocen el LPS y esto induce una vía de señalización que es dependiente de MYD88 y que conduce a la producción de RANKL por ésta célula. Pero también la unión del LPS con en TLR4 y CD14 conlleva al segundo mecanismo que es la producción de $\mathrm{PGE}_{2}$. Se conoce que la cicloxigenasa es una enzima que convierte el ácido araquidonico en Prostaglandina $\mathrm{H}_{2}\left(\mathrm{PGH}_{2}\right)$ y la prostaglandina sintasa transforma la $\mathrm{PGH}_{2}$ en $\mathrm{PGE}_{2}$, por lo tanto cuando estos receptores son activados por su ligando (LPS), se expresa en la célula una Prostaglandina sintasa de membrana que conduce a la producción de $\mathrm{PGE}_{2}$, y la $\mathrm{PGE}_{2}$ estimula la resorción ósea porque puede estimular directamente la diferenciación de osteoclastos y suprime la producción de osteoprotegerina en los osteoblastos.

Estos dos mecanismos se resumen de la siguiente manera: EI LPS es reconocido por el TLR4 y CD14 expresados en esta célula, esto induce una vía de señalización dependiente de MYD88 que conduce a un aumento en la producción de RANKL e IL-6, esta vía de señalización también permite la expresión de la cicloxigenasa y la prostaglandina sintasa de membrana lo cual conduce a la síntesis y producción de $\mathrm{PGE}_{2}$, la cual actúa de forma autocrina en la misma célula que expresa su receptor EP4 conllevando a una aumento en la producción de AMP cíclico lo cual disminuye la producción de OPG por el osteoblasto(21).

El tercer mecanismo se da gracias a la expresión del TLR4 y CD14 por los pre-osteoclastos y los osteoclastos diferenciados. Una vez estos receptores son activados por el LPS se inicia una vía que conduce a la expresión del Factor de transcripción maestro NFATc1, dando lugar a la activación, diferenciación y supervivencia de los osteoclastos ${ }^{(11,22)}$.

El cuarto mecanismo tiene que ver con el estímulo de la producción de citoquinas pro-inflamatorias, como la IL-6 por el macrófago, una vez reconoce el LPS por el TLR4 y CD14(11,21).

Otro mediador bacteriano importante es el Peptidoglican el cual es un polímero de carbohidratos cruzado por péptidos que forma parte estructural de la pared celular tanto de bacterias Gram-positivas como de Gram-negativas. El muramil dipeptido (MDP) es la mínima unidad estructural y funcional del peptidoglican responsable de inducir respuesta inmune en el huésped.

El muramil dipeptido MDP también actúa en el osteoblasto induciendo un aumento en la producción de RANKL de forma indirecta, ya que cuando el LPS es reconocido por ésta célula, se inicia una vía intracelular que también conduce a la expresión de un receptor intracelular conocido como NOD2, éste receptor reconoce el MDP y esto permite que haya una mayor producción de RANKL, por lo tanto el MDP co-estimula esta producción inducida por el LPS, lo cual conlleva a un aumento en la osteoclastogenesis (Figura 2).

Los ácidos teicoicos y lipoteicoicos son componentes de la pared celular de bacterias Gram-positivas y son factores antigénicos importantes que tienen la capacidad de desencadenar la respuesta inflamatoria por el estímulo de citoquinas y mediadores proinflamatorios. Crear un ambiente inflamatorio hace propicio el inicio de la resorción ósea, como se explicará con mas detalle más adelante.

Otros mediadores osteolíticos bacterianos son las Proteínas de choque térmico 60 de $A$. actinomycetemcomitans, colagenasas de Porphyromonas gingivalis, proteasas de otros periodontopatógenos, polisacáridos capsulares y proteínas fimbrilares ${ }^{(11,21)}$.
Tabla 1. Mediadores moleculares implicados en la pérdida de hueso periodontal.

\begin{tabular}{|c|c|c|c|}
\hline \multicolumn{2}{|c|}{$\begin{array}{c}\text { Mediadores } \\
\text { Osteolíticos Bacterianos }\end{array}$} & \multicolumn{2}{|c|}{$\begin{array}{c}\text { Mediadores } \\
\text { Osteolíticos Inmunológicos }\end{array}$} \\
\hline $\begin{array}{l}\text { LPS } \\
\text { (Lipopolisacarido) }\end{array}$ & $\begin{array}{l}\text { - Aumenta la expresión de } \\
\text { RANKL por los osteoblastos. } \\
\text { - Estimula la producción de } \\
\text { PGE }_{2} \text { por el osteoblasto. } \\
\text { - Estimula directamente la } \\
\text { diferenciación y activación } \\
\text { de los osteoclastos. } \\
\text { - Estimula la producción de } \\
\text { citoquinas pro-inflamatorias. }\end{array}$ & $\begin{array}{l}\text { Linfocitos } \\
\text { T Activados }\end{array}$ & - Producción de RANKL. \\
\hline Peptidoglican & $\begin{array}{l}\text { - Aumenta la expresión } \\
\text { de RANKL por los } \\
\text { osteoblastos. } \\
\text { - Estimula la producción de } \\
\text { citoquinas pro-inflamatorias. }\end{array}$ & $\begin{array}{l}\text { Linfocitos } \\
\text { B Activados }\end{array}$ & - Producción de RANKL. \\
\hline $\begin{array}{l}\text { Ácidos Teicoicos y } \\
\text { Lipoteicoicos }\end{array}$ & $\begin{array}{l}\text { - Estimula la producción de } \\
\text { citoquinas pro-inflamatorias. }\end{array}$ & $\begin{array}{l}\text { Monocitos/ } \\
\text { Macrofagos }\end{array}$ & $\begin{array}{l}\text { Expresan } \\
\text { RANKL } \\
\text { involucrados en } \\
\text { reclutamiento de células } \\
\text { precursoras de médula } \\
\text { ósea. } \\
-\quad \text { TNF- } \alpha \text { producida } \\
\text { por monocitos locales } \\
\text { RANKL+ promueve } \\
\text { osteoclastogenesis. } \\
\text { - Pueden desarrollarse } \\
\text { en osteoclastos al } \\
\text { encontrarse con LTCD4+ } \\
\text { activados. }\end{array}$ \\
\hline $\begin{array}{l}\text { Proteínas de choque } \\
\text { térmico } 60 \text { de } A \\
\text { actinomycetemcomitans }\end{array}$ & $\begin{array}{l}\text { - Efecto osteolítico. } \\
\text { - Estimula la producción de } \\
\text { citoquinas pro-inflamatorias. }\end{array}$ & $\begin{array}{l}\text { Células } \\
\text { Dendriticas }\end{array}$ & $\begin{array}{l}\text { - Células dendríticas } \\
\text { maduras contribuyen } \\
\text { indirectamente en la } \\
\text { osteoclastogenesis. } \\
\text { - Células dendríticas que se } \\
\text { diferencian en osteoclastos } \\
\text { DDOC. }\end{array}$ \\
\hline $\begin{array}{l}\text { Colagenasas de } \\
\text { Porphyromonas } \\
\text { gingivalis }\end{array}$ & $\begin{array}{l}\text { - Degradan colágeno Tipo } \\
\text { I de la matriz extracelular. }\end{array}$ & IL-1 & $\begin{array}{l}\text { - Estimula producción de } \\
\text { RANKL por osteoblastos } \\
\text { contribuendo a la } \\
\text { diferenciación de osteoclastos. }\end{array}$ \\
\hline $\begin{array}{l}\text { Proteasas de } \\
\text { periodontopatógenos }\end{array}$ & $\begin{array}{l}\text { - Degradan proteinas de } \\
\text { matriz extracelular. }\end{array}$ & TNF- $\alpha$ & $\begin{array}{l}\text { - Estimula producción de } \\
\text { RANKL por osteoblastos } \\
\text { contribuendo a la } \\
\text { diferenciaciónde osteoclastos. }\end{array}$ \\
\hline Polisacáridos capsulares & $\begin{array}{l}\text { - Estimula la producción de } \\
\text { citoquinas pro-inflamatorias. }\end{array}$ & $\begin{array}{l}\text { IFN-Y } \\
\text { IL-6 } \\
\text { IL-17 } \\
\text { IL-15 }\end{array}$ & $\begin{array}{lr}\text { - Efecto indirecto en } \\
\text { osteoclastogenesis por } \\
\text { activar células inmunes } \\
\text { involucradas en la } \\
\text { secreción de RANKL. }\end{array}$ \\
\hline Proteínas fimbrilares & $\begin{array}{l}\text { - Estimula la producción de } \\
\text { citoquinas pro-inflamatorias. }\end{array}$ & & \\
\hline
\end{tabular}

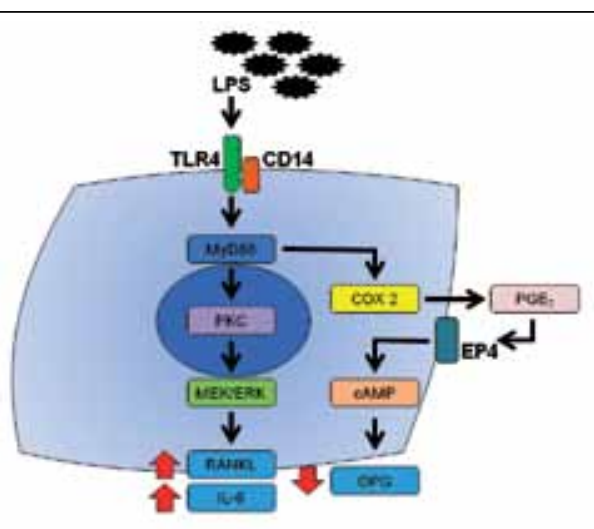

Figura 1. Inducción en la producción de RANKL, IL-6 y $\mathrm{PGE}_{2}$ por osteoblastos estimulados por el LPS bacteriano a través de TLR4 y su correceptor CD14 por la vía dependiente de MYD88. En la gráfica se observa como la $\mathrm{PGE}_{2}$ actua de forma autocrina en la misma célula induciendo una disminución en la producción de OPG. 


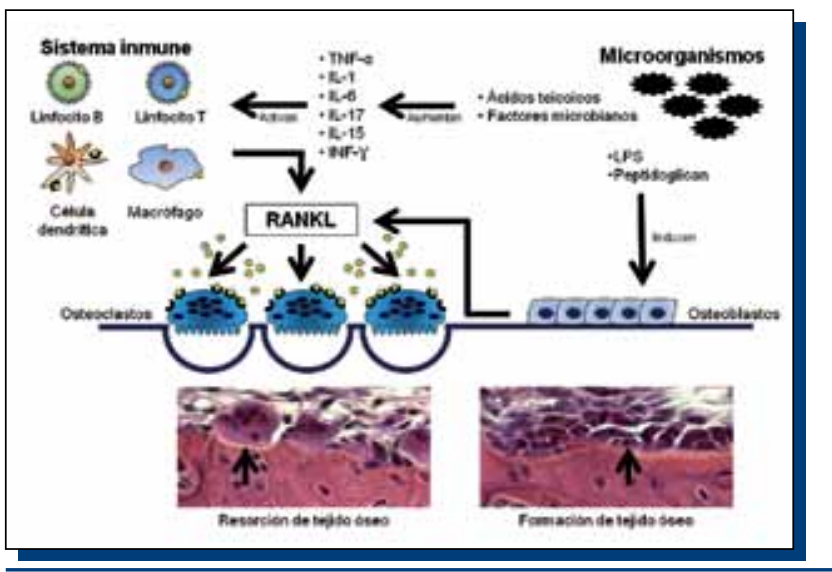

Figura 2. Mecanismos osteolíticos en la enfermedad periodontal (Modificado de: Boyle W, Simonet W, Lacey D. Osteoclast differentiation and activation. Nature, 2003; 423: 337-342 - Referencia 13).

Células Inmunes y Citoquinas Involucradas en la Destrucción de Hueso Alveolar (Tabla 1) (Figura 2)

Los microorganismos periodontopatógenos estimulan inicialmente una respuesta inmune innata la cual estimula la respuesta inmune adaptativa. Esta respuesta inmune mas especifica, especializada y adaptada cumple un papel protector pero también destructor. El linfocito TCD4+ puede diferenciarse hacia diferentes perfiles, Th1, Th2, Th17 y Tregs según el ambiente de citoquinas que se generan por el estímulo antigénico. El LPS y otros componentes bacterianos inducen la secreción de citoquinas proinflamatorias que inducen la fenotipificación hacia el linfocito Th1 cuya respuesta involucra citoquinas pro-inflamatorias como son IFN- $\gamma$ y TNF- $\alpha$, y hacia el linfocito Th17 cuya respuesta involucra citoquinas como IL-17, IL-21, IL-23 y por ésta condición están involucradas en la resorción ósea de modo indirecto al activar la respuesta inflamatoria, activando células de la respuesta inmune innata como son el macrófago y el neutrófilo respectivamente. Sin embargo, en enfermedad periodontal se han aislado también citoquinas del perfil Th2 y Tregs, lo cual puede contribuir al control de la enfermedad contrarrestando los efectos destructivos del perfil Th1 y Th17 $7^{(23,24,25)}$

Dutzan et al. en 2009 toman biopsias de tejido gingival y analizan la expresión de IFN-y y el factor de transcripción maestro del perfil Th1 (T-bet) por medio de RT-PCR encontrando una sobreexpresión de esta citoquina y del factor de transcripción T-bet, concluyendo una mayor prevalencia del perfil Th1 en la enfermedad periodontal activa ${ }^{(26)}$, así mismo Dutzan et al. en 2011 toman biopsias de tejido periodontal en 10 pacientes con periodontitis crónica y 8 pacientes sanos y analizan la expresión de citoquinas como IL-21, IL-1 $\beta$, IL-6, IL-17, IL-23, IL-10 y TGF- $\beta 1$ por medio de RT-PCR, encontrando una significativa sobreexpresión de citoquinas como IL-21, IL-1 1 , IL-6, IL-17 e IL-23 en los pacientes con periodontitis crónica en comparación con los tejidos de los pacientes sanos. La sobreexpresión de IL-21 está por lo tanto correlacionada con los parámetros clínicos de destrucción periodontal y dado que esta citoquina controla la diferenciación del linfocito $T$ hacia el perfil Th17, se muestra como este perfil prevalece en pacientes con enfermedad activa ${ }^{(27)}$.

Estos estudios muestran como los dos perfiles proinflamatorios Th1 y Th17 están implicados en la progresión y severidad de la enfermedad periodontal.

Además, una vez activado, el Linfocito TCD4+ específico de antígeno expresa y secreta RANKL, estimulando directamente la resorción ósea ${ }^{(28)}$.

La respuesta inmune humoral es también activada por el estímulo antígenico de los periodontopatógenos, cuando el Linfocito B interactúa con el Linfocito TCD4+, se induce un cambio de isotipo principalmente hacia la IgG, además éste Linfocito $B$ activado también expresa y secreta RANKL.

Dentro del surco gingival se forma una biopelícula bacteriana, los microorganismos presentes en ella, gracias a sus factores de virulencia activan la respuesta inmune innata, la cual activa una respuesta inmune adaptativa que está a cargo de los Linfocitos $T$ y Linfocitos B. Los Linfocitos TCD4+ y Linfocitos B activados expresan y secretan RANKL abundantemente, activando la osteoclastogenesis lo cual conlleva a una mayor activación de la pérdida de hueso alveolar ${ }^{(22,29,30)}$

Varios estudios han sido realizados respaldando éste juicio, como el estudio In Vitro de Kawai et al. en 2006 ${ }^{(31)}$, donde se comprueba por medio de RT-PCR una alta expresión de RANKL soluble en tejidos gingivales de pacientes con periodontitis, obtenidos después de procedimientos quirúrgicos en comparación con pacientes sanos, además en estas muestras, se analizaron los Linfocitos $B$, Linfocitos $T$ y monocitos del infiltrado celular de los tejidos gingivales por microscopia confocal, encontrando que estas células expresan y producen RANKL y además al conteo celular son mayor en número en los pacientes enfermos que en los pacientes sanos.

Dutzan et al., en 2009, en un estudio In Vitro, encuentran que en pacientes con enfermedad periodontal activa hay una sobreexpresión de Linfocitos Th17 activados secretando IL-17 y RANKL ${ }^{(32)}$.

Los monocitos/macrófagos pueden estimular la resorción ósea por su papel en la inflamación pero también algunas de estas células expresan RANKL y están involucradas en el reclutamiento de células precursoras de osteoclastos de médula ósea, producen TNF- $\alpha$, la cual promueve la osteoclastogénesis y pueden diferenciarse hacia osteoclastos al interactuar con Linfocitos TCD4+ activados ${ }^{(11,30)}$.

Las células dendríticas son muy importantes y claves dentro de la respuesta inmune, existen células dendríticas que maduran y activan la respuesta inmune adaptativa y de este modo contribuyen con la osteoclastogénesis, sin embargo hay evidencia en la literatura que existen células dendríticas que pueden diferenciarse directamente hacia osteoclastos y son denominadas DDOC (Osteoclastos Derivados de Células Dendríticas) (Figura 1).

Las células dendríticas se derivan de línea mieloide o linfoide. Las células dendríticas que vienen de línea mieloide tienen un progenitor común con osteoclastos y macrófagos. Este progenitor se diferencia hacia una célula dendrítica inmadura por la acción de citoquinas como GM-CSG e IL-4, ésta célula dendrítica inmadura se encuentra distribuida ampliamente en varios tejidos del cuerpo, actuando como un centinela esperando su encuentro con el antígeno, una vez ésta célula lo encuentra, madura y cumple funciones inmunes activando la respuesta inmune adaptativa. Sin embargo, esta célula dendrítica inmadura dentro de un ambiente inflamatorio interactúa con M-CSF y RANKL y se diferencia directamente hacia un osteoclasto( ${ }^{(30)}$.

En un estudio In Vitro realizado por Rivollier et al. en $2004^{(33)}$, se comprueba la diferenciación de monocitos extraídos de sangre periférica de pacientes adultos voluntarios, en células dendríticas tras la adición de GM-CSF e IL-4 al cultivo celular por seis días, y la diferenciación de éstas células dendríticas en osteoclastos funcionales después de adicionar M-CSF y RANKL al cultivo por 12 días, evidenciando la plasticidad de las células dendríticas y la diferenciación en osteoclastos en ambientes ricos en éstas citoquinas.

Las citoquinas también tienen un papel importante en la osteoclastogénesis, las que pertenecen al grupo de citoquinas proinflamatorias estimulan este proceso $^{(34)}$

El osteoblasto expresa receptores para la IL-1 y para el TNF- $\alpha$, éstas citoquinas aumentan durante los procesos inflamatorios y actúan en ésta célula induciendo un aumento en la producción de RANKL, éstas dos citoquinas también actúan directamente en el osteoclasto activando el factor de transcripción NF-K $\beta$ y el factor de transcripción maestro NFATc1, por lo tanto es claro su rol en la diferenciación, activación y supervivencia de los osteoclastos.

Otras citoquinas proinflamatorias como son IFN- $\gamma^{(35)}$, IL-6, IL17, IL-15 también estimulan de forma indirecta la osteoclastogénesis por estimular otras células inmunes implicadas en el aumento en la producción de RANKL.

Las citoquinas que pertenecen al grupo de citoquinas antinflamatorias como son IL-10 o TGF- $\beta$ actúan inhibiendo el proceso de osteoclastogénesis, tanto directa como indirectamente. Directamente IL-10 actúa inhibiendo la expresión de NFATc1 y TGF- $\beta$ actúa atenuando la señalización RANKL-RANK e induce un aumento en la expresión de OPG, indirectamente éstas citoquinas al inhibir el proceso inflamatorio e inactivar las células inmunes también inhíben la resorción ósea ${ }^{(11,36)}$

Tratamientos Dirigidos a Inhibir la Pérdida de Hueso Alveolar En enfermedad periodontal se han empezado a utilizar medicamentos enfocados a inhibir la pérdida de hueso alveolar actuando 
en la regulación del eje RANKL-RANK/OPG. Por lo tanto existen medicamentos que usan diferentes blancos terapéuticos, como son: el osteoclasto, RANKL y el osteoblasto.

\section{Tratamientos que Usan el Osteoclasto como Blanco Terapéutico:}

\section{a. Calcitonina}

La calcitonina es una hormona natural con una potente actividad antiresortiva debido a que el osteoclasto expresa receptores de calcitonina, una vez son activados estos receptores se inicia una vía de señalización dependiente de AMP cíclico. En respuesta a calcitonina los osteoclastos se retraen del hueso y cesa su actividad antiresortiva. A corto plazo tiene un efecto potente, pero es limitado debido a que los osteoclastos tienden a resistir a la acción de la calcitonina debido a la disminución en la expresión de los receptores y así evade éste efecto inhibitorio y se reactiva el proceso de reabsorción.

La carencia de actividad sostenida excluye la aplicación local de calcitonina como tratamiento contra la resorción ósea(37).

\section{b. Bisfosfonatos}

Son una droga antiresortiva potente. Su estructura química está compuesta por dos grupos fosfatos, que por su naturaleza proporcionan una alta afinidad por la fase mineral del hueso, y una cadena lateral $\mathrm{R}$ que le da la toxicidad al medicamento. Los bisfosfonatos tienen un efecto prolongado ya que pueden mantenerse adheridos al tejido óseo por años ${ }^{(38)}$.

De acuerdo a la naturaleza química de la cadena lateral se dividen en no nitrogenados y nitrogenados. Los no nitrogenados fueron los primeros en desarrollarse y su mecanismo de acción se dirigía a la inactivación del osteoclasto por inhibición del metabolismo de ATP en la célula, estos son el clodronato y etidronato. Los nitrogenados se desarrollaron recientemente, estos interfieren con la enzima farnesilpirofosfato sintetasa, la cual es muy importante para el ciclo celular del osteoclasto, por lo tanto esta inhibición conduce a la apoptosis del mismo, medicamentos como el zoledronato, risedronato y alendronato ${ }^{(37)}$.

Los bisfosfonatos pueden administrarse por vía oral o intravenosa y son utilizados con éxito para el tratamiento de osteoporosis u osteopenia y metástasis ósea por cáncer.

En los pacientes que reciben altas dosis por vía intravenosa pueden presentar osteonecrosis de mandíbula cuando son sometidos a procedimientos de cirugía oral, esto puede ser debido a que los bisfosfonatos se adhieren por tiempo prolongado a la fase mineral del hueso y además de actuar inactivando el osteoclasto, en el osteoblasto también actúan, lo cual hace que el hueso se convierta en un tejido acelular disminuyendo su capacidad de regeneración.

También se ha observado éste efecto en pacientes que toman bisfosfonatos por vía oral aunque en menor proporción ${ }^{(39)}$.

En enfermedad periodontal se han utilizado con éxito para la inhibición de la resorción del hueso alveolar, una vía de administración local sería ideal pero se ha descartado debido a la posibilidad de efectos $\operatorname{adversos}^{(37)}$.

\section{c. Inhibidores de Cathepsina K}

\section{d. Inhibidores Interacción Integrina av $\beta 3$ y Secuencias Arginina, Lisina y Aspartato (RGD)}

El inhibir estos últimos mecanismos solo inactiva parcialmente al osteoclasto debido a que ésta célula posee múltiples mecanismos y hormonas, antes descritos, para garantizar su función resortiva, por lo tanto no son candidatos para inhibir la pérdida de hueso alveolar en la enfermedad periodontal ${ }^{(37)}$.

\section{Tratamientos que Usan a RANKL como Blanco Terapeútico:}

\section{a. Osteoprotegerina}

Debido a que éste receptor soluble inhibe naturalmente la interacción entre RANKL-RANK, muchos autores inicialmente pensaron que la administración de osteoprotegerina podría ser una muy buena opción de tratamiento, por lo tanto se aisló la proteína y se encontró que tiene dos dominios, un dominio de unión a RANKL y otro dominio de unión al heparan sulfato, debido a que el heparan sulfato está ampliamente distribuido en todos los tejidos del cuerpo humano, al administrar la osteoprotegerina ésta se unía al heparan sulfato y no alcanzaba a llegar hasta el hueso, por lo tanto se desarrolló una proteína sintética a la cual se le cambió el dominio de unión al heparan sulfato por un dominio fc para la lgG, y con base en éstos estudios se desarrollaron dos constructos OPG-fclgG y RANK-fclgG, pero éstos inducen una respuesta inmunoreactiva, por lo tanto entraron en desuso.

Actualmente se ha desarrollado un medicamento denominado Denosumab, el cual es un anticuerpo monoclonal anti RANKL que actúa de forma similar a la OPG inhibiendo la interacción RANKL-RANK ${ }^{(37)}$.

\section{Tratamientos que Usan el Osteoblasto como Blanco Terapeútico:}

\section{a. Proteínas Wnt}

El osteoblasto produce unas proteínas denominadas Wnt, que son factores de crecimiento encargados del mantenimiento de la masa ósea. Existen unos inhibidores naturales de éstas proteínas como son Esclerostin, Frizzle y Dickkopf 1 y 2 (DKK1 y DKK2), los cuales aumentan en los procesos de resorción ósea. Inhibir estos inhibidores podría ser una buena estrategia de tratamiento. Actualmente se ha investigado un inhibidor tipo Frizzle denominado SFRP1 para el tratamiento de la resorción ósea en la periodontitis, al inhibirlo se logró una significativa disminución en la pérdida de hueso alveolar en ratas de experimentación con enfermedad periodontal inducida. Este medicamento se encuentra en fase I de investigación ${ }^{(40)}$.

\section{b. Proteínas Morfogénicas de Hueso BMP}

Estas proteínas, miembros de la familia de TGF- $\beta$, son producidas por los osteoblastos y tiene como función la inducción de células precursoras mesenquimales, por lo tanto pueden estimular la neo- formación de tejido óseo. Las más comunes son BMP-2 y BMP7. Estas proteínas no son un buen candidato para el tratamiento de la pérdida de hueso alveolar en la enfermedad periodontal puesto que generan anquilosis alrededor del diente ${ }^{(41)}$.

\section{CONCLUSIONES}

- $\quad$ El mantenimiento de la masa ósea está fuertemente regulada por la interacción específica dos tipos de células especializadas del hueso: Osteoblastos y osteoclastos.

- Los microorganismos periodontopatógenos tienen múltiples mecanismos que inducen la diferenciación y activación de osteoclastos. - La respuesta inmune inducida por los microorganismos y mediada por el huésped está involucrada en la destrucción del hueso alveolar en la enfermedad periodontal.

- La significancia clínica del eje RANKL-RANK/OPG en la enfermedad periodontal ha sido demostrada en varios estudios.

- $\quad$ El fino balance de la relación RANKL/OPG es un factor críticamente determinante que puede cambiar de salud periodontal a enfermedad periodontal.

- $\quad$ Se deben explorar mecanismos para controlar efectivamente este balance In Vivo, como futuras terapéuticas en intervenciones periodontales.

\section{CONFLICTOS DE INTERÉS}

Los autores declaran no tener conflictos de interés. 


\section{REFERENCIAS BIBLIOGRÁFICAS}

1. Nakagawa I, Amano A, Kimura R, Nakamura T, Kawabata S, Hamada S. Distribution and molecular characterization of Porphyromonas gingivalis carrying a new type of fima gene. J Clin Microbiol, 2000; 38(5): 1909-1914.

2. Socransky SS, Hafajee AD. Dental biofilms: Difficult therapeutic targets. Periodontol 2000, 2002; 28: 12-55.

3. Sbordone L, Bortolaia C. Oral microbial biofilms and plaque-related diseases: Microbial communities and their role in the shift from oral health to disease. Clin Oral Invest, 2003; 7: 181-188.

4. Armitage GC. Development of a classification system for periodontal diseases and conditions. Ann Periodontol, 1999; 4: 1-6.

5. República de Colombia, Ministerio de la Protección Social. Tercer Estudio Nacional de Salud Bucal ENSAB III. Lito Servicios. Colombia: 1999 p 99-108.

6. Demmer R, Papapanou P. Epidemiologic patterns of chronic and aggresive periodontitis. Periodontol 2000, 2010; 53: 28-44

7. Page R, Offenbacher S, Schroeder H, Seymour G, Kornman K. Advances in the pathogenesis of periodontitis: Summary of developments, clinical implications and future directions. Periodontol 2000, 1997; 14: 216-248.

8. Zhang J, Sun X, Xiao L, Xie C, Xuan D, Luo G. Gene polymorphisms and periodontitis. Periodontol 2000, 2011; 56: 102-124.

9. Yasuda H, Shima N, Nakagawa N, Yamaguchi K, Kinosaki M, Goto M, Mochizuki S, Tsuda E, Morinaga T, Udagawa N, Takahashi N, Suda T, Higashio K. A novel molecular mechanism modulating osteoclast differentiation and function. Bone, 1999; 25(1): 109-113.

10. Raggatt L, Partridge NC. Cellular and molecular mechanisms of bone remodeling. J Biol Chem, 2010; 285(33): 25103-25108.

11. Liu YC, Lerner U, Teng YTA. Cytokine responses against periodontal infection Protective and destructive roles. Periodontol 2000, 2010; 52: 163-206.

12. Fernández I, Alobera MA, del Canto M, Blanco L. Physiological bases of bone regeneration I. Histology and physiology of bone tissue. Med Oral Patol Oral Cir Bucal, 2006; 11: 47-51.

13. Boyle W, Simonet W, Lacey D. Osteoclast differentiation and activation. Nature, 2003: 423: 337-342

14. Takahashi N, Koide M, Noguchi T, Suda T. Current paradigms of osteoblastosteoclast interactions and bacterial pathogen-induced bone resorption in periodontal medicine and systems biology. Henderson B, Curtis M, Seymour R Donos N. (editors). United Kingdom 2009. P 379-393.

15. Li Z, Kong K, Qi W. Osteoclast and its roles in calcium metabolism and bone development and remodeling. Biochem Bioph Res Co, 2006; 343: 345-350.

16. Clarke B. Normal bone anatomy and physiology. Clin J Am Soc Nephrol, 2008; 3: 131-139.

17. Ferrer Cañabate J, Tovar I, Martínez P. Osteoprotegerina y sistema RANKL/ RANK: ¿El futuro del metabolismo óseo? An Med Interna, 2002; 19: 385-388.

18. Datta H, Ng W, Walker J, Tuck S, Varanasi S. The cell biology of bone metabolism. J Clin Pathol, 2008; 61: 577-587.

19. Darveau R. Periodontitis: A polymicrobial disruption of host homeostasis. Nat Rev Microbiol, 2010; 8: 481-490.

20. Paramonov N, Aduse-Opoku J, Hashim A, Rangarajan M, Curtisy M. Structura analysis of the core region of o-lipopolysaccharide of Porphyromonas gingivalis from mutants defective in o-antigen ligase and o-antigen polymerase. $J$ Bacteriol, 2009; 191(16): 5272-5282.

21. Henderson B, Nair S. Bacterial osteolytic mediators in periodontal medicine and systems biology. Henderson B, Curtis M, Seymour R, Donos N. (editors). United Kingdom 2009. P 395-405.

22. Kajiya M, Giro G, Taubman M, Han X, Mayer M, Kawai T. Role of periodonta pathogenic bacteria in RANKL-mediated bone destruction in periodontal disease. $J$ Clin Microbiol, 2010; 2: 5532-5548.

23. Botero J. Respuesta inmune en las enfermedades del periodonto: Desde la salud hasta la enfermedad y sus implicaciones terapéuticas. Rev Fac Odontol Univ Antioq, 2009; 1(1): 122-128.
24. Page R, Offenbacher S, Schroeder H, Seymour G, Kornman K. Advances in the pathogenesis of periodontitis: Summary of developments, clinical implications and future directions. Periodontol 2000, 1997; 14: 216-248.

25. Garlet G. Destructive and protective roles of cytokines in periodontitis: A reappraisal from host defense and tissue destruction viewpoints. J Dent Res, 2010; 89(12): 1349-1363.

26. Dutzan N, Vernal R, Hernandez M, Dezerega A, Rivera O, Silva N, Aguillon J Puente J, Pozo P, Gamonal J. Levels of interferon-gamma and transcription facto t-bet in progressive periodontal lesions in patients with chronic periodontitis. $J$ Periodontol, 2009; 80(2): 290-296.

27. Dutzan N, Vernal R, Vaque J, García J, Hernandez M, Abusleme L, Dezerega A, Gutkind J, Gamonal J. Interleukin-21 expression and its association with proinflammatory cytokines in untreated chronic periodontitis patients. J Periodontol, 2011; 82(10): 1483-1489.

28. Vernal R, Chaparro A, Dutzan N, Chandía S, Puente J, Silva A, Gamonal J. Asociación de mayores niveles de RANKL y Linfocitos TCD4+ en periodontitis. Revista de Estomatología, 2004; 12(1): 5-23.

29. Gemmell E, Yamazaki K, Seymour G. The role of T cells in periodontal disease: Homeostasis and autoimmunity. Periodontol 2000, 2007; 43: 14-40.

30. Andy Y, Teng T. Immune cell involvement in periodontal bone loss in periodontal medicine and systems biology. Henderson B, Curtis M, Seymour R, Donos N (editors). United Kingdom 2009. P 407-426.

31. Kawai T, Matsuyama T, Hosokawa Y, Makihira S, Seki M, Karimbux M, Goncalves R, Valverde P, Dibart S, Li Y, Miranda L, Ernst C, Izumi Y, Taubman M. B and T lymphocytes are the primary sources of RANKL in the bone resorptive lesion of periodontal disease. Immunopathology and Infectious Diseases, 2006; 169(3): 987-998.

32. Dutzan N, Gamonal J, Silva A, Sanz M, Vernal R. Over-expression of forkhead box P3 and its association with receptor activator of nuclear factor-kappa B ligand, interleukin (IL)-17, IL-10 and transforming growth factor-beta during the progression of chronic periodontitis. J Clin Periodontol, 2009; 36(5): 396-403.

33. Rivollier A, Mazzorana M, Tebib J, Piperno M, Aitsiselmi T, Rabourdin-Combe C, Jurdic P, Servet-Delprat C. Immature dendritic cell transdifferentiation into osteoclasts: Anovel pathway sustained by the rheumatoid arthritis microenvironment. Blood, 2004; 104(13): 4029-4037.

34. Chun Y, Lerner L, Teng Y. Cytokine responses against periodontal infection: Protective and destructive roles. Periodontol 2000, 2010; 52: 163-206.

35. Ayon E, Ukay T, Yokoyama M, Kishimoto T, Yoshinaga Y, Hara Y Locally administered interferon-c accelerates lipopolysaccharide-induced osteoclastogenesis independent of immunohistological RANKL upregulation. $J$ Periodontal Res, 2011; 46: 361-373

36. Graves D, Li J, Cochran D. Inflammation and uncoupling as mechanisms of periodontal bone loss. Crit Rev Oral Biol M, 2001; 90(2): 143-153.

37. Dunstan $C$. How can periodontal bone loss be stoped in periodontal medicine and systems biology. Henderson B, Curtis M, Seymour R, Donos N. (editors). United Kingdom 2009. P 427-437.

38. Tipton D, Seshul B, Dabbous M. Effect of bisphosphonates on human gingival fibroblast production of mediators of osteoclastogenesis: RANKL, osteoprotegerin and interleukin-6. J Periodontal Res, 2011; 46: 39-47.

39. Ficarra G, Beninati F, Rubino I, Vannucchi A, Longo G, Tonelli P, Prato G. Osteonecrosis of the jaws in periodontal patients with a history of bisphosphonates treatment. J Clin Periodontol, 2005; 32: 1123-1128.

40. Li C, Amar S. Inhibition of SFRP1 reduces severity of periodontitis. J Dent Res, 2007; 86(9): 873-877.

41. Kwon D, Bennett W, Herberg S, Bastone P, Pippig S, Rodriguez N, Susin C Wikesjo U. Evaluation of an injectable rhGDF-5/PLGA construct for minimally invasive periodontal regenerative procedures: A histological study in the dog. J Clin Periodontol, 2010; 37: 390-397. 